\title{
Paludismo importado por inmigrantes
}

\section{Malaria imported by immigrants}

\author{
J. Gascón i Brustenga
}

\section{RESUMEN}

Los inmigrantes de zonas endémicas que viven en Europa son los que más riesgo tienen de adquirir malaria durante los viajes a sus países, ya que suelen viajar durante más tiempo que el resto de viajeros y a zonas y en condiciones de más riesgo. Las cifras de los años 2002-2004 en España indican que $P$. falciparum es la especie más diagnosticada. África es el continente de donde se importan más casos y los inmigrantes contribuyen con el $34-41 \%$ de todos ellos.

Palabras clave. Plasmodium falciparum. Paludismo. Inmigración.

An. Sist. Sanit. Navar. 2006; 29 (Supl. 1): 121-125.

\begin{abstract}
The immigrants from endemic zones who live in Europe are those with the greatest risk of acquiring malaria during trips to their countries, since they usually travel for longer than other travellers and to zones and under conditions of greater risk. The figures for the years 2002-2004 in Spain indicate that $P$. falciparum is the most diagnosed species. Africa is the continent from which most cases are imported and immigrants contribute $34-41 \%$ of all cases.
\end{abstract}

Key words. Plasmodium falciparum. Malaria. Immigration.
Secció Medicina Tropical. Centre de Salut Internacional. Hospital Clinic de Barcelona. Barcelona.

\author{
Correspondencia: \\ Joaquim Gascón i Brustenga \\ Secció Medicina Tropical \\ Centre de Salut Internacional \\ Hospital Clinic de Barcelona \\ C/ Villarroel, 170 \\ 08036 Barcelona \\ e-mail: jgascon@clinic.ub.es
}




\section{INTRODUCCIÓN}

El paludismo o malaria es una infección causada por protozoos intracelulares del género Plasmodium. La enfermedad se transmite a través de la picadura de las hembras de mosquito del género Anopheles. Se calcula que causa entre 300 y 500 millones de casos/año con una mortalidad alrededor de 1,5 millones de personas. Las zonas endémicas ocupan más de 100 países de África, Asia, Oceanía, Oriente Medio, América Latina y algunas islas del Caribe. El 90\% de las cifras citadas corresponden al continente africano.

\section{ETIOLOGÍA}

Existen cuatro tipos de Plasmodium que afectan al hombre: $P$. falciparum, $P$. malariae, $P$. ovale y $P$. vivax. Estas cuatro especies desarrollan un ciclo evolutivo complejo, constituido esencialmente: a) por una fase asexuada en el hombre que a su vez tiene dos componentes: la fase exoeritrocitaria y la fase eritrocitaria, y b) por una fase sexuada que tiene lugar en mosquitos del género Anopheles. La fase asexual o esquizogonia se inicia cuando los esporozoítos introducidos por la picadura del mosquito alcanzan el hígado y se replican en los hepatocitos (esquizogonia exoeritrocitaria). Los merozoítos liberados de los esquizontes hepáticos pasan al torrente sanguíneo y penetran en los hematíes donde se alimentan de la hemoglobina y maduran por distintas fases hasta destruir el hematíe huésped. Al quedar libres, invaden de nuevo a otros hematíes prosiguiendo así el ciclo. La progresión del número de plasmodios es logarítmica. Estos ciclos (esquizogonia hemática) tienen una duración de 48 h para $P$. falciparum, $P$. vivax $y P$. ovale y de $72 \mathrm{~h}$ para $P$. malariae. Después de unos cuantos ciclos, junto a los esquizontes hemáticos aparecen formas sexuadas (gametos) los cuales al ser aspirados por la picadura de un Anopheles, inician el ciclo sexuado en el mosquito.

Además de esta línea común, los esporozoítos de $P$. vivax y $P$. ovale desarrollan una segunda forma hepática llamada hipnozoíto que puede permanecer de forma latente en los hepatocitos durante períodos más largos. Si no se tratan adecuada- mente, son los responsables de recidivas clínicas.

\section{EPIDEMIOLOGÍA}

La situación del paludismo en las zonas endémicas es muy variada. Mientras África Subsahariana y las Islas Salomón muestran incidencias muy altas, en otras zonas la situación de la malaria no es uniforme y varía según regiones y zonas urbanas.

España fue declarada zona libre de malaria en el año 1964. Después de unos años en los que apenas se declararon casos de malaria, el diagnóstico de paludismo vuelve a ser frecuente en nuestro país debido al auge de los viajes (turismo, negocios, cooperación, migraciones). Así, en España se declaran alrededor de 400 casos anuales de paludismo importado. En Europa los casos importados oscilan entre 13.000 y 16.000 por año con una mortalidad del 2-3\% ${ }^{1}$. En Europa también se han detectado casos del llamado paludismo de aeropuerto (casos de malaria en personas que no han viajado y que han sido picados cerca de aeropuertos, por mosquitos infectados transportados por aviones procedentes de países endémicos) o del paludismo de las maletas (mosquitos infectados atrapados en las maletas de los viajeros que son liberados al abrirse las maletas una vez han llegado a su destino). Sin embargo, ambas formas son testimoniales.

Los casos de malaria declarados en TropNet (Red Europea de Vigilancia Epidemiológica), corresponden a $P$. falciparum (78\% de los casos), P. vivax (12\%), P. ovale (4\%) y $P$. malariae (3\%). En el año 2005 , el $32 \%$ de los afectados eran inmigrantes. Las cifras de los años 2002-2004 en España indican que $P$. falciparum es la especie más diagnosticada (85\% de los casos diagnosticados, sin tener en cuenta las infecciones mixtas), seguida de $P$. vivax (12\%). P. malariae y $P$. ovale contribuyen con el 1 y $2 \%$ respectivamente. Los inmigrantes contribuyen con el $34-41 \%$ de los casos. África es, en estos momentos, la zona que exporta más malaria con el $85 \%$ de todos los casos diagnosticados en España en los últimos años. Además, $P$. falciparum es la especie predominante en los viajeros afec- 
tados que vuelven de África. La segunda especie más frecuente es $P$. vivax y es diagnosticada con más frecuencia en personas provenientes del Sudeste Asiático, Subcontinente Indio y América Latina.

Los inmigrantes de zonas endémicas que viven en Europa son los que más riesgo tienen de adquirir malaria durante los viajes a sus países de origen, ya que suelen viajar durante más tiempo que el resto de viajeros (exceptuando los cooperantes) y lo hacen a zonas y en condiciones de más riesgo.

\section{INMUNIDAD}

Algunas personas presentan rasgos genéticos que confieren cierta inmunidad contra la malaria (ausencia de antígeno Duffy en África, necesario para que $P$. vivax pueda infectarlas; hemoglobina $\mathrm{S}$ para $P$. falciparum). Pero la más importante es la inmunidad adquirida. Las sucesivas infecciones con diferentes cepas de plasmodios confieren una semi-inmunidad que provoca una disminución del número y gravedad de las crisis palúdicas. Clásicamente se ha pensado que una vez que una persona abandona una zona endémica, esta inmunidad adquirida declina a lo largo de los años hasta desaparecer. Sin embargo, un estudio reciente sugiere la persistencia de la inmunidad adquirida contra $P$. falciparum en inmigrantes de zonas endémicas después de años de estancia en Europa ${ }^{2}$.

\section{CUADRO CLÍNICO}

Después de un período de incubación que oscila entre 7-30 días (pero que puede durar meses en algunos casos de $P$. vivax y $P$. ovale) se inicia la clínica al invadir los merozoitos provenientes del hígado los eritrocitos. La clínica consiste en cefalea, malestar general, mialgias, escalofríos e intensa sensación de frío. Posteriormente, aparecen agujas febriles con rubefacción facial (período febril). El período de lisis se caracteriza por gran sudación, abatimiento, somnolencia y descenso de la temperatura. La presencia de diarrea y otros síntomas gastrointestinales, que ocurren con cierta frecuencia en el paludismo, puede provocar errores diagnósticos.
Este cuadro puede verse alterado por la aparición de complicaciones, más frecuentes cuanto más se tarda en hacer un diagnóstico y tratamiento correctos. $P$. falciparum es la especie que provoca más complicaciones (paludismo cerebral, hipoglicemia, trastornos de la coagulación, insuficiencia renal, edema agudo de pulmón,...)

Otras variables que pueden modificar la gravedad del cuadro clínico son la existencia de inmunidad previa o la toma de quimioprofilaxis.

\section{DIAGNÓSTICO DEL PALUDISMO}

En una zona no endémica como es España, la sospecha de paludismo es clave para la rapidez de su diagnóstico y su tratamiento. Por ello, una buena historia epidemiológica en los pacientes que presentan fiebre es esencial. Una simple pregunta puede dar la pista adecuada: ¿ha viajado usted recientemente a algún país del trópico? Para el paciente inmigrante será también importante saber el país de origen, el tiempo transcurrido desde la inmigración, la existencia de episodios previos de paludismo y tener en cuenta que la clínica, si existe cierto grado de inmunidad, puede presentarse de forma más larvada.

Actualmente existen diversos métodos diagnósticos que pueden utilizarse según la experiencia del profesional encargado de hacer el diagnóstico. La gota gruesa y la extensión sanguínea permiten la visualización del parásito, su cuantificación y un diagnóstico de especie. La técnica del naranja de acridina o QBC no mejora la sensibilidad del procedimiento, pero ofrece mayor precisión en la determinación de especies y en el diagnóstico de infecciones mixtas. Las técnicas de PCR son útiles en la detección de parasitemias muy bajas pero no están al alcance de muchos hospitales. Las técnicas basadas en la captura de antígeno pueden ayudar también en algunos casos o cuando no se dispone de un microbiólogo experto.

Los métodos serológicos tienen valor para estudios epidemiológicos en áreas endémicas. 


\section{TRATAMIENTO}

Una vez hecho el diagnóstico, el tratamiento no puede demorarse. El tratamiento de la malaria en los inmigrantes no difiere del que se utiliza en el resto de viajeros (Tabla 1). En los casos de paludismo por $P$. vivax, $P$. ovale y $P$. malariae, el fármaco de elección para el tratamiento de la crisis aguda es la cloroquina (dosis total de 25 $\mathrm{mg} / \mathrm{kg}$ de cloroquina base; distribuido en tres días y dando la mitad de la dosis el primer día en dos tomas separadas). En los casos de $P$. vivax y $P$. ovale, el tratamiento debe continuar con la primaquina para erradicar los hipnozoítos hepáticos y evitar así las recidivas. Es obligatoria la determinación previa de la G6PD para evitar el riesgo de anemia hemolítica farmacológica. La dosis de primaquina es de $15 \mathrm{mg} /$ día durante dos semanas. Sin embargo, se han descrito cepas de $P$. vivax, que han recidivado a pesar del tratamiento con esta dosis de primaquina ${ }^{3,4}$. Ello ha conllevado que para paludismos por $P$. vivax provenientes de ciertas zonas, la dosis utilizada sea la de 6 $\mathrm{mg} / \mathrm{kg}$ (dosis total), a razón de $30 \mathrm{mg} /$ día.

Existen cepas de $P$. falciparum resistentes a cloroquina en prácticamente todas las áreas geográficas (excepto, de momento, en América Central y Caribe). Por ello ante un caso de malaria por $P$. falciparum proveniente de América Central o Caribe, podemos utilizar también la cloroquina, a las mismas dosis descritas.

Sin embargo, para el resto de casos provenientes de Sudamérica, África, Asia, Pacífico, $P$. falciparum debe tratarse con otros fármacos. En España la combinación de fármacos más utilizada es la quinina+doxiciclina. Actualmente se dispone también de atovacuona+paludrine y de mefloquina (existen resistencias a mefloquina en cepas del sudeste asiático).

El único tratamiento disponible que se puede administrar vía endovenosa es la quinina+doxiciclina y es el tratamiento de elección en los casos graves o en los que la vía oral no es posible (vómitos). En caso de embarazo, la doxiciclina debe sustituirse por clindamicina.

\section{PROFILAXIS ANTIPALÚDICA}

La toma de medicamentos durante los viajes a zonas endémicas es una de las estrategias más utilizadas por los viajeros para evitar padecer malaria. Otras medidas importantes son el uso de repelentes y sobre todo el buen uso de mosquiteras impregnadas. En la tabla 2 se recogen las pautas quimioprofilácticas más utilizadas.

Tabla 1. Combinaciones de fármacos antipalúdicos más usadas España.

\begin{tabular}{|c|c|c|}
\hline Fármacos antipalúdicos & Dosis más utilizadas & Duración del tratamiento \\
\hline $\begin{array}{l}\text { Atovacuona }(\mathrm{A})+\text { proguanil }(\mathrm{P}) \\
\text { (combinación fija) }\end{array}$ & $\begin{array}{l}\text { Adultos: } 1 \mathrm{~g}(\mathrm{~A})+400 \mathrm{mg}(\mathrm{P}) / \mathrm{d} \\
\text { Niños: entre } 250 \mathrm{mg}(\mathrm{A})+100 \mathrm{mg}(\mathrm{P}) \\
\text { y } 1 \mathrm{~g}(\mathrm{~A})+400 \mathrm{mg}(\mathrm{P}) \text { según peso } \\
\text { al día. }\end{array}$ & 3 días \\
\hline Cloroquina* & $\begin{array}{l}\text { Cloroquina*: } 25 \mathrm{mg} / \mathrm{kg} \text { dosis total } \\
\text { ( } 10 \mathrm{mg}+5 \mathrm{mg} \text { a las } 6 \text { horas } \\
\text { el primer día; } 5 \mathrm{mgr} \text { el segundo día } \\
\text { y } 5 \mathrm{mg} \text { el tercer día) }\end{array}$ & 3 días \\
\hline Mefloquina & $\begin{array}{l}\text { Mefloquina: } 15 \mathrm{mg} / \mathrm{kg} \text { o } 25 \mathrm{mg} / \mathrm{kg} \\
(15 \mathrm{mg}+10 \mathrm{mg})\end{array}$ & $\begin{array}{l}\text { Dosis única o dos dosis } \\
\text { (a las } 6 \text { o 24h) }\end{array}$ \\
\hline Quinina + clindamicina & $\begin{array}{l}\text { Quinina**: } 10 \mathrm{mg} / \mathrm{kg} / 8 \mathrm{~h} \\
\text { (máx. en adultos: } 600 \mathrm{mg} / 8 \mathrm{~h} \text { ) } \\
\text { Clindamicina }: 10 \mathrm{mg} / \mathrm{kg} / 12 \mathrm{~h}\end{array}$ & $\begin{array}{l}\text { Quinina: } 7 \text { días } \\
\text { Clindamicina: } 5 \text {-7 días }\end{array}$ \\
\hline Quinina + doxiciclina & $\begin{array}{l}\text { Quinina**: } 10 \mathrm{mgr} / \mathrm{kg} / 8 \mathrm{~h} \\
\text { (máx.: } 600 \mathrm{mg} / 8 \mathrm{~h} \text { ) } \\
\text { Doxiciclina: } 200 \mathrm{mg}+100 \mathrm{mg} / \mathrm{d} \\
\text { (niños: dosis según peso) }\end{array}$ & $\begin{array}{l}\text { Quinina: } 7 \text { días } \\
\text { Doxiciclina: } 7 \text { días }\end{array}$ \\
\hline
\end{tabular}


Tabla 2. Pautas más recomendadas de profilaxis antipalúdica.

\begin{tabular}{|c|c|c|c|}
\hline Fármaco & $\begin{array}{l}\text { Dosis } \\
\text { adultos }\end{array}$ & $\begin{array}{l}\text { Dosis } \\
\text { pediatría }\end{array}$ & Duración \\
\hline Cloroquina & $300 \mathrm{mg} /$ semana & $5 \mathrm{mg} / \mathrm{kg} / \mathrm{semana}$ & $\begin{array}{l}\text { Desde } 1 \text { semana antes } \\
\text { del viaje hasta } 4 \text { semanas } \\
\text { después }\end{array}$ \\
\hline $\begin{array}{l}\text { Cloroquina (C ) + } \\
\text { paludrine (P) }\end{array}$ & $\begin{array}{l}\text { C: mismas dosis } \\
\text { P: } 200 \mathrm{mg} / \mathrm{d}\end{array}$ & $\begin{array}{l}\text { C: mismas dosis } \\
\text { P: } 3 \mathrm{mg} / \mathrm{kg} / \text { día }\end{array}$ & $\begin{array}{l}\text { Como cloroquina sola: } \\
\text { desde } 1 \text { día antes del } \\
\text { viaje hasta } 4 \text { semanas } \\
\text { después }\end{array}$ \\
\hline $\begin{array}{l}\text { Cloroquina + paludrine } \\
\text { (dosis fijas) }\end{array}$ & $\begin{array}{l}100 \mathrm{mg} / \text { dia de } \mathrm{C} \\
+200 \mathrm{mg} / \mathrm{d} \text { de } \mathrm{P}\end{array}$ & & $\begin{array}{l}\text { Desde } 1 \text { día antes del } \\
\text { viaje hasta } 4 \text { semanas } \\
\text { después }\end{array}$ \\
\hline Mefloquina & $250 \mathrm{mg} /$ semana & $5 \mathrm{mg} / \mathrm{kg} / \mathrm{semana}$ & $\begin{array}{l}\text { Desde } 2-3 \text { semanas antes } \\
\text { del viaje hasta } 4 \text { semanas } \\
\text { después }\end{array}$ \\
\hline Doxiciclina & 100 mg/día & $\begin{array}{l}1,5 \mathrm{mg} \text { de } \mathrm{sal} / \mathrm{kg} \\
\text { al día }\end{array}$ & $\begin{array}{l}\text { Desde } 1 \text { día antes hasta } 4 \\
\text { semanas después del } \\
\text { viaje }\end{array}$ \\
\hline $\begin{array}{l}\text { Atovacuona + proguanil } \\
\text { (dosis fijas) }\end{array}$ & $250 \mathrm{mg}+100 \mathrm{mg}$ al día & $\begin{array}{l}\text { 11-20 kg: } 1 \text { compr } \\
\text { pediátrico/día } \\
21-30 \mathrm{~kg}: 2 \text { compr } \\
\text { pediátricos/día } \\
31-40 \mathrm{~kg}: 3 \text { compr } \\
\text { pediátricos/día } \\
\text { >40 kg: dosis de adulto }\end{array}$ & $\begin{array}{l}\text { Desde } 1 \text { día antes hasta } 1 \\
\text { semana después del } \\
\text { viaje. }\end{array}$ \\
\hline
\end{tabular}

\section{Agradecimientos}

Agradezco a la Dra. Luisa P. Sánchez del Centro Nacional de Epidemiologia ISCIII, Madrid la información epidemiológica de la malaria importada en España.

\section{BIBLIOGRAFÍA}

1. LEGROS F, DANIS M. Surveillance of malaria in European countries. Eurosurveillance 1998; 3: 45-47.

2. Bouchaud O, Cot M, Kony S, Durand R, SchieMann R, Ralaimazava $P$ et al. Do African immi- grants living in France have long-term malarial immunity? Am J Trop Med Hyg 2005; 72: 21-25.

3. DoherTy JF, DAY JH, WARHURST DC, ChIODINI PL. Treatment of Plasmodium vivax malariatime for a change? Trans Roy Soc Trop Med Hyg 1997; 91: 76.

4. Muñoz J, Velasco M, Valls ME, Corachán M., GASCÓN J. ¿Cuánta primaquina para los hipnozoitos de plasmodium vivax? Enf Infecc Microbiol Clin 2006; 24: 29-30. 
